III JOURNÉES DE FORMATION DES ENSEIGNANTS D'ÉDUCATION PHYSIQUE : RENCONTRES CAPAS-Cité

Tarbes, le 13 de février 2019

\title{
Promotion de l'activité physique pour la santé à l'école : formation théorique et pratique
}

\section{Registre des comptes rendus}

Coordonnateurs:

Alberto Aibar Solana

Raquel Pérez-Ordás

Sergio Estrada Tenorio 



\section{JOURNÉES DE FORMATION DES ENSEIG- NANTS D'ÉDUCATION PHYSIQUE : RENCONTRES CAPAS-Cité}

Tarbes, le 13 de février 2019

\section{Promotion de l'activité physique pour la santé à l'école : formation théorique et pratique}

\section{Registre des comptes rendus}

\section{CRÉDITS}

(C) Alberto Aibar Solana, Raquel Pérez-Ordás et Sergio Estrada Tenorio

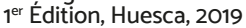

Edité par: CAPAS- Cité/CAPAS-Ciudad. Universidad de Zaragoza

ISBN : ISBN 978-84-16723-66-9

Imprimé par : Universidad de Zaragoza. Tous droits réservés. Toute forme de reproduction, distribution, publication ou modification de cette ceuvre est interdite sans l'autorisation de ses auteurs, sauf exception prévue par la loi. Veuillez vous adresser à (www.

cedro.org) pour photocopier ou scanner une partie de l'œuvre.

Coordonnateurs:

Alberto Aibar Solana

Raquel Pérez-Ordás

Sergio Estrada Tenorio

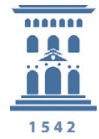

Servicio de

Publicaciones

Universidad Zaragoza 


\section{Sommaire}

PÁGE

INTRODUCTION : « Promotion de l'activité physique pour la santé à l'école :

formation théorique et pratique»

\section{VOLET : L'ACTIVITÉ PHYSIQUE À L'ÉCOLE : CONTEXTUALISATION}

7. Les niveaux d'AP des enfants et des adolescents en général, à l'école et en EPS. Léna Lhuisset, Julien Bois, Caroline Bernal y Nicolas Fabre (CAPAS-Cité, Université de Pau et des Pays de l'Adour)

12. La promotion de l'AP pour la santé en EPS en France. Carole Sève (Ministère de l'éducation nationale, France)

15. La promotion de l'AP pour la santé en EPS en Espagne. José Antonio Julián y Alberto Aibar (Université de Zaragoza)

\section{VOLET : ACTIONS ET RÉSULTATS D’EXPÉRIENCES}

20. Cours théorique en activité au collège de Lourre Barrousse. Ludovic Dolce et Magalie Annecy (Collège Loures Barousse, EPS)

24. Exemple d'innovation pédagogique : le travail collaboratif : Effet d'un dispositif d'apprentissage coopératif sur le bien-être psychosocial des élèves. Pascal Legrain et Guillaume Escalie (STAPS, Université de Bordeaux)

26. Intervention en milieu scolaire pour promouvoir l'activité physique et diminuer le temps sédentaire chez une population d'enfants de quartiers défavorisés de 6-11 ans.

Caroline Bernal, Nicolas Fabre, Léna Lhuisset et Julien Bois. (CAPAS-Cité, Université de Pau et des Pays de l'Adour)

\section{VOLET : ATELIERS PRATIQUES}

29.

Les « breaks » de sédentarité Organisation de la classe pour diminuer le temps sédentaire Activités « actives » dans la classe.

Caroline Bernal et Nicolas Fabre (Université de Pau et des Pays de l'Adour)

33. Exemple de mise en place du travail collaboratif.

Pascal Legrain et Guillaume Escalie (UF STAPS, Université de Bordeaux)

34. Théorie et pratique sur la promotion d'AP : Rencontres inter-centres. "Le Test de Responsabilité". Une stratégie avant les Rencontres inter-centres. Paz Tierz, Gemma Bermejo, Raquel Pérez et Eduardo Generelo (CAPAS- Cité)

37. Théorie et pratique sur le transport actif : Rencontres inter-centres. José Antonio Julián, Javier Sevil y Alberto Aibar (Université de Zaragoza)

40. Réflexions sur l'organisation des situations d'enseignement en E.P.S. ayant un impact sur la santé : le travail intermittent à haute intensité en sports collectifs. Laurent Souriac (Université de Pau et des Pays de l'Adour) 


\title{
"Promotion de l'activité physique pour la santé à l'école : formation théorique et pratique".
}

\author{
Introduction :
}

La troisième édition des Journées de formation des enseignants CAPAS-Cité à Tarbes vient clore une série de rencontres de formation destinées aux enseignants en vue de renforcer le réseau de formation permanente des professeurs existant au niveau transpyrénéen. Le projet Capas-Cité (Centre pyrénéen pour la promotion de l'activité physique et de la santé) vise à promouvoir l'activité physique et les modes de vie sains afin d'améliorer la qualité de vie de la population. Les III Journées de formation des enseignants intitulées " Promotion de l'activité physique pour la santé à l'école : formation théorique et pratique " se sont tenues à Tarbes en février 2019 et souhaitent promouvoir l'amélioration de la qualité de vie de la population par le biais d'une rencontre où les expériences pratiques exposées reflètent les aspects théoriques qui sont examinés au niveau national et international. L'esprit transpyrénéen de cette action repose sur les relations des acteurs de la promotion de l'activité physique entre la France et l'Espagne qui collaborent et travaillent ensemble depuis ces dernières années.

Les objectifs des III Journées de formation des enseignants étaient essentiellement les suivants :

- Promouvoir une meilleure connaissance sur l'état actuel de la promotion de l'activité physique en milieu scolaire.

- Diffuser les connaissances relatives aux stratégies efficaces de promotion de l'activité physique dans les établissements scolaires.

- Enclencher des réflexions entre des acteurs de différents pays sur la manière la plus efficace d'aborder la promotion de l'activité physique et de la santé.

Des professeurs d'établissements scolaires, d'éducation physique en formation initiale ainsi que les différents acteurs du système éducatif qui sont chargés de la promotion de la santé des élèves (personnel de gestion, école d'infirmières, etc.) ont participé à cette formation.

Différentes communications, expériences et ateliers en rapport avec le thème principal de la journée ont été présentés et organisés en trois grands volets :

1. L'ACTIVITÉ PHYSIQUE À L'ÉCOLE : CONTEXTUALISATION

2. ACTIONS ET RÉSULTATS D'EXPÉRIENCES

3. ATELIERS PRATIQUES 
Les communications relatives au thème principal ont été présentées dans le volet " l'activité physique à l'école ", sous les intitulés suivants :

1. Les niveaux d'AP des enfants et des adolescents en général, à l'école et en EPS.

2. La promotion de l'AP pour la santé en EPS en France.

3. La promotion de l'AP pour la santé en EPS en Espagne.

Les travaux suivants qui concernent des expériences pratiques appliquées ont été présentés dans le volet "Actions et résultats d'expériences » :

1. Cours théorique en activité au collège de Lourre Barrousse.

2. Exemple d'innovation pédagogique : le travail collaboratif : Effet d'un dispositif d'apprentissage coopératif sur le bien-être psychosocial des élèves.

3. Intervention en milieu scolaire pour promouvoir l'activité physique et diminuer le temps sédentaire chez une population d'enfants de quartiers défavorisés de 6-11 ans.

Pour clore les journées, le volet «Ateliers pratiques » a été proposé autour des thèmes suivants :

1. Les "breaks » de sédentarité Organisation de la classe pour diminuer le temps sédentaire Activités " actives " dans la classe.

2. Exemple de mise en place du travail collaboratif.

3. Théorie et pratique sur la promotion d'AP : Rencontres inter-centres. "Le Test de Responsabilité". Une stratégie avant les Rencontres inter-centres.

4. Théorie et pratique sur le transport actif : Rencontres inter-centres.

5. Réflexions sur l'organisation des situations d'enseignement en E.P.S. ayant un impact sur la santé : le travail intermittent à haute intensité en sports collectifs.

Le présent document souhaite recueillir sous forme de compte rendu et de manière synthétique l'ensemble des travaux et des contributions académiques présentés dans les différents volets des III Journées de formation des enseignants. Nous espérons qu'ils serviront d'outils de soutien aux enseignants et aux professionnels chargés de la promotion de l'activité physique qui désirent se recycler, découvrir les méthodes d'autres professionnels et en définitive, continuer à se former dans la promotion de l'activité physique pour l'amélioration de la santé.

Coordonnateurs du document

Alberto Aibar Solana, Raquel Pérez-Ordás y Sergio Estrada Tenorio 


\section{Les niveaux d'AP des enfants et des adolescents en général, à l'école et en EPS.}

Julien Bois', Léna Lhuisset', Caroline Bernal' y Nicolas Fabre' 'Université de Pau et des Pays de l'Adour, Laboratoire Mouvement Equilibre Performance Santé (EA 4445).

\section{Introduction :}

L'objectif de cette communication est de présenter l'état des connaissances sur les niveaux d'activité physique et de sédentarité des enfants et des adolescents. Ces données sont présentées pour une journée complète puis détaillées pour le contexte spécifique de l'école et enfin dans celui du cours d'éducation physique. Lorsque cela est possible les données mondiales sont d'abord présentées, puis les données spécifiques à la France et enfin les données locales que nous avons pu observer dans les études conduites sur Tarbes et sa région, ou dans le cadre de collaborations avec des collègues de l'université de Saragosse.

Nous rappellerons dans un premier temps les définitions des concepts clés utilisés avant de présenter les chiffres à l'échelle de la journée globale, puis dans le contexte scolaire et enfin dans le cadre spécifique du cours d'éducation physique et sportive.

\section{Définitions des termes :}

L'activité physique (AP) est définie comme « tout mouvement corporel produit par contraction des muscles squelettiques entraînant une augmentation de la dépense énergétique par rapport à la dépense énergétique de repos » (Caspersen, Powell, \& Christenson, 1985). Elle constitue la composante la plus variable de la dépense énergétique totale d'un individu. L'AP regroupe l'ensemble des activités qui peuvent être pratiquées dans différents contextes en poursuivant des objectifs variés (utilitaires, sanitaires, sociaux, etc.). Les principaux domaines de pratique d'AP sont le travail, les transports, les activités domestiques et les loisirs. Ces derniers incluent l'exercice, le sport et l'AP de loisirs non structurée.

L'intérêt d'un concept aussi large est d'envisager des changements de comportements qui ne se limitent pas aux seules activités de loisirs et aux activités organisées, mais qui puissent également s'intéresser à l'organisation du temps scolaire, du travail dans la classe ou aux modes de transports.

La sédentarité ou comportement sédentaire désigne les activités qui n'augmentent pas sensiblement la dépense énergétique au-dessus du ni- 
veau de repos et incluent des activités telles que dormir, s'asseoir, se coucher et regarder la télévision, ainsi que d'autres formes de divertissement sur écran. Afin d'évaluer l'activité physique des enfants et des adolescents il est généralement fait référence aux recommandations de l'Organisation Mondiale de la Santé $(\mathrm{WHO}, 2010)$. L'idée est de proposer des repères clairs en matière d'activité physique quotidienne bénéfique pour la santé. Autrement dit de définir le niveau d'AP quotidien minimum à adopter pour garantir une bonne santé. Ces recommandations sont formulées en terme d'activité physique modérée à vigoureuse, ce qui correspond à un seuil d'activité d'une intensité suffisante (>3 MET) pour mettre en route un certain nombre de mécanisme physiologique de notre organisme : on parle donc d'activité physique modérée à vigoureuse (APMV en français ou MVPA en anglais). L'activité physique légère n'entre pas dans l'APMV ce qui explique qu'APMV et sédentarité ne sont pas des vases communicants: supprimer du temps sédentaire ne se traduit pas nécessairement par une augmentation d'APMV car le nouveau comportement adopté peut être de l'activité physique légère.

Les principales recommandations pour les enfants et les adolescents sont présentées ci-dessous:

Pour les enfants et jeunes gens de cette classe d'âge, l'activité physique englobe notamment le jeu, les sports, les déplacements, les activités récréatives, l'éducation physique ou l'exercice planifié, dans le contexte familial, scolaire ou communautaire. Pour améliorer l'endurance cardio-respiratoire, la forme musculaire et l'état osseux et réduire le risque de maladies non transmissibles, il est recommandé ce qui suit :

1. Les enfants et jeunes gens de 5 à 17 ans devraient accumuler au moins 60 minutes par jour d'activité physique d'intensité modérée à soutenue.

2. La pratique d'une activité physique pendant plus de 60 minutes par jour apportera un bénéfice supplémentaire pour la santé.

3. L'activité physique quotidienne devrait être essentiellement une activité d'endurance. Des activités d'intensité soutenue, notamment celles qui renforcent le système musculaire et l'état osseux, devraient être incorporées, au moins trois fois par semaine. » (WHO, 2010)

Ainsi le repère pour définir si un enfant est considéré comme suffisamment actif est de 60 minutes d'activité physique. Le terme «inactif» ou «inactivité physique » caractérise un niveau insuffisant d'AP d'intensité modérée à vigoureuse (APMV) c'est-à-dire inférieur au seuil recommandé. 
Niveaux d'activité et de sédentarité pour la journée entière :

\section{Activité physique et inactivité}

Au niveau mondial 80,3\% des adolescents ne respectent pas les recommandations d'APMV, les garçons étant légèrement plus actifs que les filles (Hallal et al., 2012). Pour les enfants il est délicat d'arriver à trouver des valeurs consensuelles car les variations méthodologiques liées à la mesure de l'activité physique conduisent à des résultats très variables (Guinhouya, Samouda, \& De Beaufort, 2013).

En France l'étude ESTEBAN (Santé Publique France, 2015) qui fait référence en la matière, indique que $72 \%$ des garçons et $82 \%$ des filles n'atteignent pas les recommandations, soit un chiffre assez proche des valeurs mondiales.

Les études que nous avons conduites localement, basées sur des mesures directes par accélérométrie indiquent que $46 \%$ des enfants (Bernal et al., 2017) et $83 \%$ des adolescents (Aibar, Bois, Generelo, Zaragoza, \& Paillard, 2012) n'atteignent pas les recommandations et sont donc considérés comme insuffisamment actifs. Si l'on s'intéresse au niveau moyen d'APMV il s'élève à $65 \mathrm{mn} /$ jour et à $43 \mathrm{mn} / \mathrm{jour}$ respectivement pour les enfants et les adolescents de ces études (Aibar, et al., 2012; Bernal et al., 2017). La diminution forte et rapide des niveaux d'APMV de l'enfance à l'adolescence est bien documentée et encourage à conduire des actions de promotion dès le plus jeune âge.

\section{Sédentarité}

Les travaux sur la sédentarité sont plus délicats à synthétiser car les indicateurs fluctuent d'une étude à l'autre certains évaluant le temps passé devant les écrans, d'autres le temps passé à regarder la télévision ou le temps assis et d'autres encore mesurant le temps sédentaire total évalué par accélérométrie.

Au niveau mondial les données disponibles indiquent qu'environ 42\% des 15-29 ans déclarent passer 4 heures par jour ou plus assises (Hallal et al., 2012). En France, l'étude ESTEBAN s'est centrée sur le temps passé devant un écran (TV, jeux vidéos, travail sur l'ordinateur). Elle révèle que près de $50 \%$ de $6-10$ ans, $70 \%$ des $11-14$ ans, $71 \%$ des filles et $87 \%$ des $15-17$ ans passent 3h/jour ou plus devant un écran. En 10 ans cette durée moyenne a augmenté de 30 minutes chez les 6-10 ans, d'1 heure 15 minutes chez les 11-14 ans et de 2 heures chez les 15-17 ans (Santé Publique France, 2015).

Les études réalisées localement portent sur le temps sédentaire total, c'est-à-dire le temps passé assis ou debout avec une très faible dépense énergétique. Ces mesures réalisées par accélérométrie indiquent que chez les enfants 10 heures par jour sont en moyenne consacrées à des activités sédentaires, soit environ $79 \%$ du temps (Bernal et al., 2017). Chez les adolescents les valeurs observées indiquent 9 heures 30 minutes de temps sédentaire quotidien en moyenne (Aibar et al., 2015). 


\section{Niveaux d'activité et de sédentarité observés dans le temps scolaire :}

Les études présentant les niveaux d'activité physique et de sédentarité dans le temps scolaire sont peu nombreuses, aussi présenterons-nous les résultats issus de nos travaux. Des recommandations spécifiques existent pour l'APMV réalisée dans le contexte scolaire. II est préconisé que 50\% de l'APMV quotidienne soit réalisée dans le cadre scolaire (Pate et al., 2006), afin de faciliter le respect des 60 minutes d'APMV quotidienne recommandés.

Les données que nous avons observées chez des enfants d'école primaire indiquent que seulement 38\% de l'APMV quotidienne soit 25 minutes environ est réalisée dans le contexte scolaire (Bernal et al., 2017). Les valeurs observées chez les adolescents sont meilleures puisque environ 47,5 \% de leur APMV quotidienne est réalisée dans le contexte scolaire.

Concernant le temps sédentaire accumulé durant le temps scolaire, nous disposons seulement des données relatives aux enfants d'école primaire. Les résultats indiquent qu'environ $81 \%$ du temps scolaire des enfants est du temps sédentaire, soit 4 heures 10 minutes de temps sédentaire sur 5 heures 30 minutes de temps scolaire théorique (hors pause méridienne).

Ainsi le système scolaire d'une part peine à rendre les jeunes suffisamment actifs, les 50\% d'APMV quotidienne dans le temps scolaire étant rarement atteint, et d'autre part génère une quantité importante de temps sédentaire. Par conséquent une prise de conscience du rôle central du système scolaire dans la promotion de l'activité physique et dans la lutte contre la sédentarité semble urgente. Une modification de l'organisation de la journée à l'école, un changement des pratiques d'enseignement vers des classes plus activités, de même qu'une augmentation du volume des cours d'éducation physique sont des pistes qu'il devient urgent d'explorer.

\section{Le contexte spécifique du cours d'Éducation Physique et Sportive :}

Par définition le cours d'éducation physique est le contexte idéal pour accumuler un maximum d'APMV. II est recommandé que 50\% du temps du cours d'éducation physique soit réalisé en APMV. Les données disponibles laissent penser que cet objectif est très rarement atteint. Par exemple une étude suisse (Aibar \& Chanal, 2015) sur plus de 1200 élèves (de 10 à 12ans) révèlent qu'environ 25\% seulement du temps de séance se déroulent en APMV et 40\% environ en temps sédentaire, le reste se retrouvant en activité physique légère. Bien évidemment ces chiffres sont susceptibles de varier considérablement selon le type de séance réalisé, les activités pratiquées, la distance entre l'établissement scolaire et le lieu de pratique. Quoi qu'il en soit l'écart entre l'activité réelle des élèves et les recommandations semble là encore majeur.

\section{Conclusion :}

Les données présentées révèlent des niveaux d'APMV globalement inquiétants pour les enfants et les adolescents. Un faible niveau d'activité physique durant ces 
âges est en effet prédictif d'une activité physique insuffisante à l'âge adulte. Or l'activité physique est un facteur de prévention et de protection contre la plupart des maladies non-transmissibles telles que le diabète, l'hypertension, les maladies cardio-vasculaires, les cancers (INSERM, 2019; Warburton, Nicol, \& Bredin, 2006).

Les données présentées révèlent que dans le contexte scolaire global ou dans celui plus spécifique de l'éducation physique, les niveaux requis pour rester en bonne santé ne sont pas atteints. Or l'école constitue pour la puissance publique un moyen d'action tout à fait privilégié qui pourrait permettre d'agir dès le plus jeune âge pour le développement de saines habitudes de vie. Le constat dressé laisse penser qu'il reste encore beaucoup à faire, malgré l'intérêt majeur de ce sujet pour la santé des futures générations et la diminution des dépenses de santé auquel il pourrait conduire. Ainsi promouvoir l'activité physique et lutter contre la sédentarité dans le contexte scolaire devrait être un objectif de première importance pour les futures politiques de santé publique.

\section{Bibliographie :}

Aibar, A., Bois, J. E., Generelo, E., Zaragoza, J., \& Paillard, T. (2012). A cross-cultural study of adolescents' physical activity levels in France and Spain. European Journal of Sport Science, 13(5), 551-558. doi: 10.1080/17461391.2012.746733

Aibar, A., \& Chanal, J. (2015). Physical education: The effect of epoch lengths on children's physical activity in a structured context. PLoS ONE, 10(4). doi: 10.1371/journal.pone.0121238

Aibar, A., Bois, J. E., Zaragoza, J., Bru, N., Paillard, T., \&, Generelo, E. (2015). Adolescents' Sedentary Behaviors in Two European Cities. Research quarterly for exercise and sport, 86(3), 233-243. doi: 10.1080/02701367.2015.1039891

Bernal, C., Lhuisset, L., Fabre, N., Chanal, J., Ruelloux, J., \& Bois, J. (2017). Influence de l'activité physique et des habiletés motrices sur les capacités attentionnelles et la réussite scolaire chez des enfants d'école primaire. Paper presented at the Congrès International de l'Association des Chercheurs en Activités Physiques et Sportives (ACAPS), Dijon.

Caspersen, C. J., Powell, K. E., \& Christenson, G. (1985). Physical activity, exercise and physical fitness: definitions and distinctions for health-related research. Public Health Reports, 100(2), 126-131.

Guinhouya, B. C., Samouda, H., \& De Beaufort, C. (2013). Level of physical activity among children and adolescents in Europe: A review of physical activity assessed objectively by accelerometry. Public Health, 127(4), 301-311. doi: 10.1016/j.puhe.2013.01.020

Hallal, P. C., Andersen, L. B., Bull, F. C., Guthold, R., Haskell, W., \& Ekelund, U. (2012). Global physical activity levels: Surveillance progress, pitfalls, and prospects. The Lancet, 380(9838), 247-257. doi: 10.1016/ s0140-6736(12)60646-1

INSERM. (2019). Activité physique. Prévention et traitement des maladies chroniques. In E. Sciences (Ed.), (pp. 824).

Pate, R. R., Davis, M. G., Robinson, T. N., Stone, E. J., McKenzie, T. L., \& Young, J. C. (2006). Promoting physical activity in children and youth: A leadership role for schools - A scientific statement from the American Heart Association Council on Nutrition, Physical Activity, and Metabolism (Physical Activity Committee) in collaboration with the Councils on Cardiovascular Disease in the Young and Cardiovascular Nursing. Circulation, 114(11), 1214-1224. doi: 10.1161/circulationaha.106.177052

Pate, R. R., O'Neill, J. R., \& Lobelo, F. (2008). The evolving definition of "sedentary". Exercise and Sport Sciences Reviews, 36(4), 173-178. doi: 10.1097/JES.0b013e3181877d1a

Santé Publique France. (2015). Étude de santé sur l'environnement, la biosurveillance, l'activité physique et la nutrition (Esteban) 2014-2016. Volet nutrition. Chapitre Activité physique et sédentarité.

Warburton, D. E. R., Nicol, C. W., \& Bredin, S. S. D. (2006). Health benefits of physical activity: The evidence. CMAJ, 174(6), 801-809. doi: 10.1503/cmaj.051351

WHO (2010). Global Recommendations on Physical activity for Health. Geneva: World Health Organization. 


\title{
L'école et la promotion de la santé des élèves
}

\author{
Carole Sève ${ }^{1}$ \\ ${ }^{1}$ Inspectrice Générale de l'éducation nationale, \\ Ministère de l'éducation nationale, Paris.
}

\section{Résumé :}

L'enjeu de cette présentation est d'examiner la manière dont l'institution scolaire s'est emparée des enjeux de promotion de la santé des élèves. Elle fait un focus sur l'enseignement de l'EPS et pointe que si la santé est explicitement inscrite dans les enjeux de la discipline, dans les pratiques des enseignants d'EPS les préoccupations de santé passent souvent en arrièreplan. L'EPS seule ne peut atteindre les enjeux de santé fixés par l'institution scolaire : elle est une affaire collective devant engager l'ensemble de la communauté éducative.

\section{L’école et la promotion de la santé des élèves :}

Promouvoir la santé à l'école est un enjeu d'importance pour lutter contre les inégalités scolaires (Pinel-Jacquemin, 2016). Différents travaux montrent en effet le lien entre la santé des élèves et leur réussite scolaire. La loi de juillet 2013 sur la refondation de l'école de la République décrit sept axes principaux pour la promotion de la santé à l'école : la mise en place d'un environnement scolaire favorable à la santé, la mise en œuvre de programmes d'éducation à la santé, la participation à la politique de prévention sanitaire, la réalisation des bilans de santé, la détection des problèmes de santé, l'accompagnement des élèves, et la participation à la veille épidémiologique. Ces axes couvrent ainsi un champ très large allant de la protection à la prévention en passant par l'éduction. Plusieurs circulaires décrivent différents dispositifs pour faire vivre ces axes. Par exemple, le parcours éducatif de santé (circulaire de janvier 2016) vise à une mise en cohérence des différentes actions de promotion de la santé menées au sein d'un établissement scolaire, afin de lutter contre l'inefficacité de la multiplication d'actions ponctuelles en matière de santé mentionnée par Broussouloux et Lamoureux (2007) ; les comités d'éducation à la santé et à la citoyenneté (circulaire d'août 2016) sont chargés de la mise en place d'une éducation à la santé dans les établissements scolaires en associant différents partenaires éducatifs.

L'approche de la santé par l'institution scolaire s'appuie sur une conception globale de la santé (en référence à la définition de la santé par l'OMS) qui intègre des composantes physiologique, psychologique et sociale : elle effectue un rapprochement entre la santé, la responsabilité et la citoyenneté. 
Le parcours éducatif de santé insiste sur l'importance de la participation des élèves et de leur attitude réflexive. II s'agit de faire prendre conscience aux élèves d'un certain nombre de leurs connaissances et expériences, afin de les amener à prendre soin d'eux-mêmes et des autres, et éviter les conduites à risques.

Si l'institution scolaire insiste sur l'engagement de toute la communauté éducative pour prendre en charge la santé des élèves, dans les faits trois catégories de personnels sont essentiellement concernés : les infirmiers scolaires qui appliquent la politique de santé du ministère de l'éducation nationale et qui sont très souvent investis dans des actions d'éducation à la santé, les psychologues scolaires qui prennent en charge les souffrances psychologiques des enfants, et les enseignants dont la participation à l'éducation à la santé est inscrite dans leur référentiel professionnel. Les attitudes des enseignants vis-à-vis de l'éducation à la santé sont cependant très diverses. Gaborit (2015), dans une étude sur la participation d'enseignants d'école maternelle à un programme d'éducation à la santé, identifie quatre profils : les "enseignants bons élèves" (qui mettent en place le programme), les "enseignants débordés" (qui n'ont pas le temps de mettre en place le programme car ils ont d'autres priorités), les "enseignants réfractaires" (qui refusent le programme car il est en contradiction avec leurs propres valeurs), et les "enseignants distants" (qui ne sont pas intéressés par le programme).

Par ailleurs, lorsque l'on parle d'éducation à la santé à l'école, deux disciplines scolaires sont souvent mises en avant : les sciences de la vie et de la terre (du fait de thématiques de leur programme en relation avec la santé) et l'éducation physique et sportive. Cet appel à l'EPS tient à des représentations sociales ("le sport c'est la santé »), à des connaissances scientifiques (le lien entre activité physique et santé), et aux programmes de cette discipline dans lesquels les enjeux de santé sont inscrits explicitement. Pour les cycles 2, 3 et 4, une des compétences générales de l'EPS des programmes est "Apprendre à entretenir sa santé par une activité physique régulière ". La manière dont se décline cette compétence sur les trois cycles donne à voir une conception de la santé comme étant essentiellement la gestion de ses capacités physiques. Au lycée un objectif général de l'EPS est « Construire durablement sa santé » et articule dans sa présentation des dimensions physiologique, psychologique et sociale. Concernant les conceptions de la santé en EPS, Moralès et Travaillot (2015) distinguent deux courants principaux : (a) un courant qu'ils nomment hédoniste dans lequel l'accent est porté sur l'engagement physique, le bien-être, le plaisir, et (b) un courant qu'ils nomment appropriatif dans lequel l'accent est porté sur l'acquisition de compétences et de savoirs spécifiques (sur et pour la santé). Ces deux courants expriment deux approches différentes de la santé. Cependant dans les pratiques des enseignants d'EPS, il semble que cette préoccupation de santé des élèves passe en arrière-plan comparativement à d'autres. En effet, les études sur l'activité des enseignants d'EPS pointent de manière fréquente des préoccupations relatives au maintien de l'ordre, à la mise en activité des élèves, à l'apprentissage, au développement de leur autonomie... mais rarement à la 
santé. Par ailleurs, les enseignants d'EPS définissent de manière diverse et souvent de façon floue l'éducation à la santé (Loison, 2015). Aussi, il semble que cet enjeu de santé associé à l'EPS, relève plus d'un affichage de la discipline que de réelles stratégies d'enseignement. Intégrer des enjeux de santé dans les pratiques des enseignants d'EPS reste un défi à relever et doit s'organiser autour de plusieurs principes : une définition partagée de la santé, une conception de la santé qui prend en compte les représentations des élèves afin d'éviter une approche normalisante et moralisatrice, la formalisation de contenus d'enseignement spécifiques à la santé en fonction des besoins des élèves, la proposition de modalités de pratique favorisant un engagement physique des élèves, une organisation de la pratique pour faire vivre des expériences de bien-être perceptibles à court et moyen terme. Ceci en sachant que le volume hebdomadaire d'EPS est compris, selon les niveaux de classe, entre 2 et 4 heures, et qu'elle poursuit d'autres objectifs éducatifs. L'EPS ne peut à elle seule atteindre les objectifs d'éducation à la santé fixés par l'institution scolaire. La promotion de la santé des élèves à l'école est une affaire collective qui doit engager l'ensemble de la communauté éducative, s'inscrire dans la durée, et proposer des actions complémentaires et cohérentes à différents niveaux.

\section{Bibliographie :}

Broussouloux S., \& Lamoureux, P. (2007). Le développement de l'éducation à la santé à l'école. Situations de la France et du Québec. Les Tribunes de la santé 3(16), 61-68.

Gaborit, E. (2015). Sanitarisation de l'école et scolarisation de la santé. Socioéthnographie de la mise en oeuvre et appropriation du programme "Bien manger et bouger à la maternelle" en Haute Garonne et en Ariège. Thèse, Faculté des Sciences du Sport et du Mouvement Humain, Université Toulouse III, Toulouse. Co-dirigée par Nadine Haschar-Noé et Gérard Neyrand.

Loison D. (2015) Quels savoirs enseignés en éducation à la santé dans les séances d'EPS ? Éducation, Santé, Sociétés, 2(1), 13-31.

Morales Y., \& Travaillot Y. (2015). Débats et controverses autour des programmes d'EPS. Revue Education, Santé, Société, 2, 95-110.

Pinel-Jacquemin S. (2016). Bien-être des élèves à l'école et promotion de leur santé. Rapport commandé par le Cnesco. https://www.cnesco.fr/fr/qualite-de-vie-a-lecole/ 


\section{La promotion de l'AP pour la santé en EPS en Espagne}

José Antonio Julián ${ }^{1-2}$ y Alberto Aibar ${ }^{2-3}$

${ }^{1}$ Facultad de Ciencias Sociales y Humanas. Universidad de Zaragoza.

${ }^{2}$ Grupo de Investigación EFYPAF. Universidad de Zaragoza.

${ }^{3}$ Facultad de Ciencias Humanas y de la Educación. Universidad de Zaragoza.

\section{Résumé :}

La législation espagnole en vigueur souligne l'obligation des administrations éducatives de promouvoir la pratique de l'activité physique lors de la journée de classe. De nombreuses initiatives ont été menées par les différents ministères et les Communautés autonomes afin de doter les enseignants et les familles des établissements scolaires des ressources nécessaires à la mise en œuvre de cette promotion.

Nous présentons huit décisions que les enseignants et les établissements scolaires peuvent prendre en vue d'impliquer le plus grand nombre d'acteurs possibles et créer des ambiances saines dans chaque contexte. Enfin, nous mettons l'accent sur la responsabilité de l'administration pour mener des initiatives basées sur la preuve scientifique.

\section{Introduction :}

Le rapport entre l'éducation et la santé offre de telles possibilités d'influence bidirectionnelle qu'il ne doit pas négligé au niveau scolaire. Les deux concepts doivent aller de pair dans le processus de construction sociale des futurs citoyens qui se trouvent actuellement dans les classes de l'enseignement obligatoire. Le fait que les élèves plus sains apprennent mieux et que ces adultes mieux éduqués sont des sujets plus sains est largement connu et accepté. (Kolbe, 2019). C'est pourquoi, il est essentiel de favoriser les associations collaboratives entre les systèmes qui améliorent les niveaux de santé et d'éducation de la société dans son ensemble. L'activité physique (AP) et sa promotion qui vise à faire des citoyens alphabétisés du point de vue physique devient un élément clé de cette relation potentielle entre la santé et l'éducation. Le modèle conceptuel WSCC de I'ASCD (The Whole School Whole Community, Whole Child) peut servir d'approche théorique de référence qui réunit les objectifs communs des deux secteurs et considère l'activité physique et l'éducation physique comme un composant essentiel à travailler au sein de la communauté. 


\section{Le rôle de l'école dans la promotion de l'activité physique en Espagne :}

L'école, et plus particulièrement l'éducation physique et sportive (EPS) joue un rôle essentiel dans la construction d'un mode de vie actif et sain (Abarca-Sos, Murillo, Julián, Zaragoza, \& Generelo, 2015). Des modèles comme le Programme global d'activité physique scolaire (CSPAP; National Association for Sport and Physical Education, 2008) considèrent l'EPS comme un des éléments clés parmi les options de pratique offertes par le quotidien scolaire.

Une telle situation est renforcée par la législation espagnole en vigueur (Loi organique 8/2013) qui reconnaît l'obligation des administrations et par conséquent des établissements scolaires de promouvoir la pratique de l'activité physique lors de la journée scolaire. Pour ce faire, il existe une multitude d'initiatives hors programmes et de matériel didactique pédagogique produit par l'administration afin d'accompagner et d'aider la communauté éducative en général et les professeurs des écoles en particulier, dans leur travail de promotion de l'activité physique (par exemple, la stratégie nationale NAOS, Nutrition, Activité physique et Prévention de l'Obésité).

\section{Décisions pour la promotion de l'activité physique à l'école :}

D'après nos connaissances sur la réalité du contexte éducatif, lorsqu'il existe une inquiétude visant à encourager un mode de vie actif et sain chez les élèves, les enseignants et les établissements scolaires peuvent prendre une série de décisions afin d'impliquer le plus grand nombre d'acteurs possible et générer ainsi des environnements plus favorables à la santé. Ces décisions sont les suivantes :

1. L'organisation de l'éducation physique et sportive.

2. Optimiser la méthodologie des activités.

3. Développer des actions visant à intégrer les apprentissages de la «gestion de la vie active ".

4. Relier le contexte scolaire à la réalité sociale.

5. Développer des projets interdisciplinaires.

6. Développer un projet global de promotion de l'AP et de la santé.

7. Projets globaux de promotion de l'AP coordonnés entre les établissements.

8. Institutionnaliser et évaluer les actions qui sont réalisées dans le contexte scolaire.

Nous allons expliquer succinctement ces différentes décisions dont certaines ont déjà été recueillies par Julián, Abarca-Sos, Zaragoza, \& Aibar (2016). 
1) L'organisation de l'éducation physique et sportive. À partir de la proposition de la maîtrise de l'action motrice réalisée par Parlebas (2006) et d'autres études d'innovation éducative qui ont été menées à bien dans notre contexte, 5 blocs de contenus sont compris sous cette dénomination : Bloc 1 : actions motrices individuelles ; bloc 2 : actions motrices d'opposition ; bloc 4 : actions motrices en plein air ; bloc 5 : actions motrices à visée artistique ou expressive. Les enseignants sont invités ainsi à ce que leurs élèves reçoivent un traitement approprié dans le domaine de l'EPS tout au long de l'étape, au moyen d'un travail systématique (toutes les classes), équilibré (même nombre de séances dans les cinq premiers blocs) et contextualisé (adapté aux possibilités de l'établissement et de l'environnement) des différents types d'expériences motrices.

2) Optimiser la méthodologie des activités. II faut fournir des expériences satisfaisantes d'apprentissage avec le développement d'environnements et de motivations optimaux dans toutes les unités didactiques (Julián, 2012).

3) Développer des actions pour intégrer les apprentissages de la " gestion de la vie active ». Les apprentissages liés à la pédagogie au moyen/à partir de conduites motrices et les apprentissages transversaux sont réunis dans un bloc spécifique. Ce bloc appelé " gestion de la vie active et valeurs " souligne les différents apprentissages importants pour la discipline tels que : les règles et les principes d'intervention ; l'activité physique, les habitudes de position, d'hygiène et d'alimentation ; les effets physiologiques de la réalisation de l'activité physique liés à la santé ; l'acceptation des différentes réalités corporelles ; la reconnaissance du patrimoine culturel ; la construction de la vie active et le développement des valeurs individuelles, sociales et environnementales. L'idée qui doit prédominer en abordant ce bloc est qu'il faut le développer de manière globale avec les situations d'apprentissage que l'enseignant programmera à partir des cinq autres blocs ou au moyen d'actions spécifiques tout au long de l'année scolaire.

4) Relier le contexte scolaire à la réalité sociale. Nous devons utiliser les événements sociaux et culturels du contexte proche en rapport avec l'AP afin d'organiser nos unités didactiques de manière à ce qu'elles permettent de mettre en action tous les apprentissages qui ont été développés lors de celle-ci. De cette manière, nous donnerons une meilleure fonctionnalité aux apprentissages demandés aux étudiants.

5) Développer des projets interdisciplinaires. L'EPS recèle un grand potentiel pour la mobilisation des apprentissages scolaires car les activités proposées aux élèves constituent une situation de compétence en soi ; elles mobilisent toutes les ressources disponibles (acquis, attitudes, connaissances, adresses, etc.) dans des conditions qui sont reliées à la vie réelle des élèves. Les étudiants identifient clairement au quotidien 
la natation, la course d'endurance, le tennis, la lutte, le basket, le volley, les défis coopératifs, l'orientation, le VTT, les danses traditionnelles, l'expression corporelle, etc. ce qui lui confère un potentiel globalisateur et fédérateur dans le domaine éducatif (Julián, Ibor, Aibar, \& Aguareles, 2017). Nous recommandons la proposition de Julián et al. (2017b) pour approfondir cette idée.

6) Développer un projet global de promotion de l'AP et de la santé. II est intéressant de développer des projets globaux dans un contexte établi par les écoles promotrices de la santé pour relier les différents agents et acteurs qui ont une influence dans un contexte éducatif. Comme nous le savons, plus il y a de connexions, d'espaces et de moments qui sont intentionnellement enrichis, plus les possibilités de développer un environnement favorable à la santé se multiplient. (Sevil, García-González, Generelo, \& Aibar, 2019).

7) Projets globaux de promotion de l'AP coordonnés entre les établissements scolaires. Le fait que différentes écoles ayant des projets de promotion de l'AP similaires se trouvent à différents moments d'une étape éducative est un formidable défi pour encourager les interactions avec l'AP comme élément majeur. (Navarro et al., 2017).

Les décisions énoncées sont présentées à un niveau croissant de difficulté dû aux agents et aux acteurs qu'il faut mobiliser et impliquer pour pouvoir les mener à bien. Par ailleurs, les différents niveaux de qualité pourraient être déterminés à chaque décision afin de les optimiser.

\section{Conclusion :}

La dernière décision est d'institutionnaliser et évaluer les actions qui sont réalisées dans le contexte scolaire. Qu'est-ce qui fonctionne dans la promotion de l'AP à l'école ?, quelles conditions doivent réunir les établissements scolaires pour développer des projets transformateurs ?, quelles stratégies s'avèrent plus efficaces ? sont différentes questions auxquelles l'administration doit répondre en collaboration avec des chercheurs et des établissements scolaires. La promotion de l'AP exige le besoin d'une conduite politique claire qui incite les administrations compétentes en matière d'éducation et de santé à devenir des institutions primordiales pour la coordination, l'orientation et l'évaluation de projets basés sur la preuve scientifique dont s'alimentent à leur tour les processus de promotion de conduites saines comme l'AP. 


\section{Bibliographie :}

ASCD: www.ascd.org/learningandhealth

Abarca-Sos, A., Murillo, B., Julián, J. A., Zaragoza, J., \& Generelo, E. (2015). La Educación Física: ¿Una oportunidad para la promoción de la actividad física? Retos, 28, 155-159.

Estrategia nacional española NAOS. http://www.aecosan.msssi.gob.es/AECOSAN/web/nutricion/seccion/estrategia_naos.htm

Julián, J. A. (2012). Motivación e intervención docente en la clase de educación física. Tándem. Didáctica de la Educación Física, 40, 7-17.

Julián, J. A., Abarca-Sos, A., Zaragoza, J., \& Aibar, A. (2016). Análisis crítico de la propuesta del currículum básico de la LOMCE para la asignatura de Educación Física. Acciones derivadas en la Comunidad Autónoma de Aragón y propuestas de futuro. Retos, 29, 173-181.

Julián, J. A., Ibor, E., Aibar, A., \& Aguareles, I. (2017). Educación física, motor de proyectos. Tándem. Didáctica de la Educación Física, 56, 7-15.

Julián, J. A., Zaragoza, J., Abarca-Sos, A., Aibar, A., Tena, I., Ibor, E.,..., \& Tartaj, N. (2017). Los proyectos interdisciplinares en la formación inicial del profesorado de educación física. Aula de Innovación Educativa, 266, 12-16.

Kolbe, L. J. (2019). School health as a strategy to improve both public health and education. Annual Review of Public Health, 40(3), 1-3. https://doi.org/10.1146/annurev-publhealth-040218-043727

Ley Orgánica 8/2013, de 9 de diciembre, para la Mejora de la Calidad Educativa (LOMCE), Boletín Oficial del Estado (BOE). 10 de diciembre de 2013.

National Association for Sport and Physical Education (2008). Comprehensive school physical activity programs. Reston, VA: Author. Available at https://www.cdc.gov/healthyschools/physicalactivity/cspap.htm

Navarro, A., Martín, D., García, C., Jofre, E., Hernández, P., \& Gómez, D. (2017). Proyecto Amigos Activos. Tándem. Didáctica de la Educación Física, 55, 59-66.

Sevil, J., García-González, L. Generelo, E., \& Aibar, A. (2019). Can high schools be an effective setting to promote healthy lifestyles? Effects of a multiple behavior change intervention in adolescents. Journal of Adolescent Health, 64(4), 478-486. doi: 10.1016/j.jadohealth.2018.09.027

Parlebas, P. (2006). L'action motrice, fer de lance de l'éducation physique. Les Cahiers EPS de l'Académie de Nantes, 34, 5-9. 


\section{Actions et résultats d'expériences}

\section{Cours théorique en activité au collège de Lourre Barrousse}

Ludovic Dolce $^{1}$ y Magalie Annecy ${ }^{1}$

Collège Loures Barousse, EPS

\section{Constat à l'origine de l'action en 2012 :}

Lors de l'année scolaire 2012-2013, une réflexion a été lancée au collège de la Barousse sur l'opportunité d'ouvrir une nouvelle expérimentation " apprendre autrement » dans la continuité de l'expérimentation nationale « cours le matin, sport l'après-midi » arrivant à terme afin de permettre aux élèves de 6ème une bonne intégration dans le cycle secondaire en travaillant le sens de l'école et les méthodes d'apprentissage.

\section{1 - Objectifs poursuivis}

La mise en place de l'expérimentation vise plusieurs objectifs :

1. Faire profiter des élèves en difficulté scolaire ou à besoins éducatifs spécifiques d'une petite structure dynamique, innovante dont les effectifs par classe sont très corrects (environ 20 élèves/classe).

2. Mettre en place des rythmes scolaires propices aux apprentissages

3. Enclencher au sein de l'établissement une réflexion sur les apprentissages des élèves

4. Favoriser le travail en équipe

Nombre d'élèves et niveau $(\mathrm{x})$ concernés Les élèves de 6ème sont concernés par cette nouvelle expérimentation soit en moyenne 40 élèves

\section{2 - Description}

L'architecture actuelle des emplois du temps basée sur une adaptation des rythmes scolaires au rythme quotidien et hebdomadaire le plus favorable aux apprentissages est conservée et le travail est orienté dans une voie plus axée sur la motivation et la métacognition. On sait que les apprentissages scolaires demandent un effort aux élèves d'où le travail sur la motivation en donnant du sens aux apprentissages; on sait aussi qu'il manque souvent les méthodes d'apprentissage aux élèves. 


\section{3 - Modalités de mise en ceuvre}

Les temps dégagés dans le cadre de l'expérimentation sont notamment dévolus au travail sur ces deux axes : motivation et métacognition en faisant prendre conscience aux élèves que les connaissances et compétences acquises pendant la semaine sont transférables sur des activités pratiques le jeudi après-midi et le vendredi après-midi (temps de l'expérimentation).

Le jeudi plutôt sur des activités éducatives et culturelles, le vendredi après-midi sur des activités sportives. Le travail en équipe interdisciplinaire est indispensable pour nourrir cette expérimentation.

"Tous les vendredis sont réservés aux sorties APPN, ou l'énergétique est le pilier central des randonnées pédestres. Les 2 Classes sont prises en charge tous les vendredis de $14 \mathrm{~h}$ à 17h.Lutte contre la sédentarité, l'obésité... Les compétences sociales sont également au cœur de ces sorties sportives ».

\section{4 - Moyens mobilisés}

7 heures supplémentaires hebdomadaires permettent aux enseignants par discipline de mettre en pratique les enseignements théoriques en transdisciplinarité avec les enseignants d'EPS.

$3000 €$ par an permettent de prévoir des déplacements et des interventions de personnes extérieures à l'établissement. (L'expérimentation «cours le matin, sport l'après-midi était financée à hauteur de 500 euros).

\section{5 - Partenariat et contenu du partenariat}

Travail en partenariat avec les mairies et la communauté des communes de la Barousse du fait de la mise en application de l'expérimentation sur de nombreux sites historiques et naturels proches du collège.

\section{6 - Évaluation}

Modalités du suivi et de l'évaluation de l'action (auto-évaluation, évaluation interne, externe) : Un comité de pilotage se réunit tous les mois afin de faire le point sur l'organisation du programme et ses améliorations.

Validation du palier 2 du socle commun pour les élèves qui ne l'avaient pas validé en $\mathrm{CM} 2$.

Trois ressources ou points d'appui qui vous ont permis de progresser

- Patrimoine historique et naturel situé aux alentours du collège

- Le travail d'équipe des enseignants.

- la réussite de la première expérimentation « cours le matin, sport l'après-midi ». 
Deux difficultés éventuelles rencontrées

- L'adaptation des emplois du temps hebdomadaire.

- les moyens financiers qui ne suivent pas pour permettre les différentes sorties extérieures.

Effets constatés...

- Sur les acquis des élèves.

- Un bien être des élèves de 6ème au sein du collège.

- Cohésion entre les élèves.

- Les élèves à besoins particuliers sont moins en difficulté. Sur les pratiques des enseignants.

- Développement de la transdisciplinarité.

- Adaptation de certaines pratiques aux autres niveaux sur le leadership et les relations professionnelles

- Sur l'école / l'établissement

- Conforte son image novatrice en termes d'apprentissages plus généralement, sur l'environnement

\section{Qu'en est-il aujourd'hui....en 2018 :}

Nous avons dû faire évoluer notre dispositif (qui à l'origine était expérimental ...sur 3 ans). Aujourd'hui, APPRENDRE AUTREMENT est un dispositif qui perdure dans notre collège, mais uniquement grâce à la motivation des personnels qui sont fortement investis dans ce système. En effet depuis la fin de la période d'expérimentation, nous n'avons plus aucun moyen (financier, humain) alloués à ce dispositif. Ainsi nous fonctionnons sur les moyens propres de l'établissement.

Des disciplines ont donné des heures d'autonomie pour pouvoir fonctionner, les sorties sont souvent payantes et nécessitent des efforts de l'établissement.

Les élèves de 6ème ont donc un roulement toutes les 3 semaines pour les sorties APPN. Ils alternent avec des cours d'EMC, le prix littéraire et de l'Aide Personnalisée.

Voici les Sorties APPN et Apprendre Autrement : nous utilisons le tissu local... 


\begin{tabular}{|c|c|c|}
\hline & Matières reliées à l'EPS & Travail de la sortie \\
\hline 1 & SVT & $\begin{array}{l}\text { Classification des végétaux lors d'une } \\
\text { randonnée pédestre à Sarp } \\
\text { (20min à pieds du collège) } 3 \mathrm{~h}\end{array}$ \\
\hline 2 & $H G$ & $\begin{array}{l}\text { Étude de lecture de paysage au sommet } \\
\text { du Mont Bourg Izaourt (700m). } 4 \mathrm{~h}\end{array}$ \\
\hline 3 & EMC & $\begin{array}{l}\text { A la Mairie de Loures Barousse } \\
10 \text { min à pieds) 3h }\end{array}$ \\
\hline 4 & ED MUSICALE & $\begin{array}{l}\text { Travail sur l'orgue à St Bertrand de } \\
\text { Comminges avec l'organiste locale: } \\
\text { nous partons là-bas en rando pédestre } \\
\text { ou VTT selon l'effectif } 3 \mathrm{~h}\end{array}$ \\
\hline 5 & MATHS & $\begin{array}{l}\text { Travail mathématique sur les « arcs » à } \\
\text { travers d'une course d'orientation dans la } \\
\text { ville médiévale. } 3 \mathrm{~h}\end{array}$ \\
\hline 6 & EMC & $\begin{array}{l}\text { Randonnée raquette neige au Mourtis } \\
\text { ( } 45 \text { min en bus). } 4 \mathrm{~h}\end{array}$ \\
\hline 7 & $\begin{array}{l}\text { Liaison école / collège avec les } \\
\text { P.E des écoles du secteur } \\
\text { - St Bertrand } \\
\text { - Loures } \\
\text { - Saléchan }\end{array}$ & $\begin{array}{l}\text { Travail multidisciplinaire sur une journée : } \\
\text { Année paire : travail proportionnalité } \\
\text { + français sur le livre l'enfant océan : } \\
\text { Escalade + course d'orientation } \\
\text { Année impaire : travail à St Bertrand de } \\
\text { Comminges sur les ruines romaines, } \\
\text { français, arts plastiques et math : } \\
\text { Course d'orientation + tir à l'arc }\end{array}$ \\
\hline
\end{tabular}

Nos effectifs ne cessent d'augmenter au collège de la Barousse. Cette année il nous a été plus difficile de maintenir ce dispositif car nous n'avions que deux 6è jusqu'à présent, mais cette année nous en avons trois !!! Mais nous avons réussi, en supprimant malheureusement des sorties... 


\section{Exemple d'innovation pédagogique: le travail collaboratif : Effet d'un dispositif d'apprentissage coopératif sur le bien-être psychosocial des élèves}

Pascal Legrain ${ }^{1}$ G Gillaume Escalie ${ }^{1}$ 'UF STAPS, Université de Bordeaux

\section{Résumé:}

La récente constitution d'un cycle CM1-CM2- $6^{\text {ème }}$ appelle un changement des pratiques enseignantes afin d'assurer une continuité des apprentissages des élèves entre l'école élémentaire et le collège. La systématisation de certains " formats pédagogiques " de façon synchronique (entre les disciplines) et diachronique (tout au long du cycle) peut constituer un vecteur de stabilisation des apprentissages dans la transition inter-degrés favorable au bien-être psychosocial des élèves.

Dans une tradition socioconstructiviste des acquisitions, cette communication rend compte d'une recherche collaborative à l'échelle pluridisciplinaire visant à accompagner les enseignants d'un réseau école-collège à construire, à partir d'un modèle commun, des dispositifs d'apprentissage coopératif innovants et à les mettre en œuvre en classe. Du point de vue méthodologique, des données quantitatives visant à examiner les effets de l'implémentation de ce type de dispositifs sur des dimensions psychosociales liées au bien-être des élèves ont été couplées à des données qualitatives analysant l'impact de la mise en œuvre de ces dispositifs sur l'activité des enseignants en classe.

D'une part, les résultats mettent en évidence des effets significatifs de ce type de dispositifs sur la motivation autodéterminée des élèves, ou encore sur la moindre évolution de l'anxiété sociale. D'autre part, ils soulignent toute la nécessité de considérer la mise en œuvre de ces dispositifs dans leurs dimensions à la fois génériques et disciplinaires. 


\section{Bibliographie :}

Aronson, E., \& Patnoe, S. (1997). The jigsaw classroom: Building cooperation in the classroom. New York: Addison-Wesley.

Cohen, E. G. (1994). Restructuring the classroom: Conditions for productive small groups. Review of Educational Research, 64, 1-35.

Gillet, N., Rosnet, E., \& Vallerand, R. J. (2008). Development of a scale to assess basic need satisfaction in the sport context. Canadian Journal of Behavioural Science, 40, 230-237.

Gillet, N., Vallerand, R. J., Amoura, S., \& Baldes, B. (2010). Influence of coaches' autonomy support on athletes' motivation and sport performance: A test of the hierarchical model of intrinsic and extrinsic motivation. Psychology of Sport and Exercise, 11, 155-161.

Johnson, D. W., \& Johnson, R. T. (2014). Cooperative learning in 21th century. Anales de Psicología, 30, 841-851.

Vallerand, R. J., Blais, M. R., Brière, N. M., \& Pelletier, L. G. (1989). Construction et validation de l'Échelle de Motivation en Éducation (EME). Revue canadienne des sciences du comportement, 21, 323-349. 


\title{
Intervention en milieu scolaire pour promouvoir l'activité physique et diminuer le temps sédentaire chez une population d'enfants de quartiers défavorisés de 6-11 ans
}

\author{
Caroline Bernal', Nicolas Fabre', Léna Lhuisset ${ }^{1}$ Y Julien Bois ${ }^{1}$ \\ 1 Université de Pau et des Pays de l'Adour \\ Laboratoire « Mouvement, Equilibre, Performance et Santé »
}

\section{Résumé :}

Avec l'avènement de l'ère technologique, nous assistons à une diminution de l'activité physique (AP) et une augmentation du temps sédentaire (TS) des enfants. Il a été constaté dans deux écoles primaires situées dans un quartier défavorisé sur Tarbes que les enfants passent en moyenne 10 heures en position sédentaire et $37 \%$ des enfants sont également inactifs. Une intervention de 11 mois a été mise en place dans les deux écoles concernées. Diverses stratégies sont utilisées pour promouvoir l'activité physique et impliquent les enfants, leurs familles, l'école, la communauté et les politiques publiques (Bronfenbrenner, 1977).

\section{Contexte :}

L'activité physique (AP), se définissant comme tout mouvement produit par les muscles squelettiques entrainant une augmentation de la dépense énergétique possède de nombreux bienfaits sur la santé physique, mentale et sociale des enfants (Grélot, 2016). Celle-ci aurait également des répercussions positives sur les capacités cognitives des enfants, et notamment sur les capacités d'attention, d'inhibition et de flexibilité mentale (Donnelly et al, 2016). L'OMS recommande aux enfants de pratiquer 60 minutes d'activité physique modérée et vigoureuse par jour, dont 30 minutes sur le temps scolaire. La sédentarité se définit comme un ensemble de comportements qui requièrent une dépense énergétique très faible $(<1,5 \mathrm{MET})$ en position assise et de repos (Barnes et al., 2012). L'OMS recommande de diminuer autant que possible l'accumulation de temps sédentaire tous les jours.

\section{Étude :}

Cette étude est réalisée dans deux écoles primaires situées en « Réseau d'Education Prioritaire » de la ville de Tarbes : l'école Jean-Jacques Rousseau ainsi que l'école Jules Verne. Des mesures initiales en Novembre 2016 et en Mai 2017 ont été effectuées et ont permis d'évaluer les niveaux 
d'activité physique et de sédentarité des enfants, ainsi que le développement des principales capacités attentionnelles des enfants. Nous avons constaté que $37 \%$ des enfants ne respectent pas les recommandations de l'OMS et qu'ils cumulent plus de 10 heures de temps sédentaire par jour. $80 \%$ du temps entre $12 \mathrm{~h}$ et $14 \mathrm{~h}$ est passé assis.

A la suite de ces mesures, une intervention pour promouvoir l'activité physique a été mise en place à l'école Jean Jacques Rousseau (située en REP) pendant l'année scolaire 2017/2018, pour tous les niveaux scolaires (du CP au CM2). Des mesures ont été effectuées pendant et après cette intervention, afin de voir si elle a été efficiente. L'intervention est également en cours à l'école Jules Verne pendant l'année scolaire 2018/2019.

Les niveaux d'AP et de sédentarité des enfants peuvent dépendre de nombreux facteurs, qui, dans le modèle socio-écologique, peuvent se trouver dans les différentes sphères : personnelle (psychologie), interpersonnel (amis, parents), organisationnelle (école), communautaire (quartier, ville) et politique (programmes « santé » mis en place) (Bronfenbrenner, 1977).

Divers leviers d'interventions ont été mis en place dès cette année en lien avec le modèle socio-écologique: :

1. Aménagement de la cour de récréation : des traçages ont été réalisés dans la cour.

2. Aménagement du service de cantine : le temps passé à la cantine entre $12 \mathrm{~h}$ et $14 \mathrm{~h}$ a été réduit afin de libérer du temps de jeu pour l'enfant avant les cours de l'après-midi.

3. Ateliers de sensibilisation pour les enfants : ils sont effectués en classe et en récréation, afin de leur faire prendre conscience de la nécessité d'une hygiène de vie optimale.

4. Ateliers de sensibilisation pour les parents : ils visent également une prise de conscience de l'importance des comportements de santé et abordent des aspects plus pratiques concernant l'activité physique et la sédentarité de l'enfant.

5. Formations pour les enseignants : elles concernent tous les niveaux scolaires. Au total, 4 réunions de 2 heures ont été prévues, et diverses problématiques sont abordées. a) Des temps de dialogue permettant de réfléchir sur les notions de comportements de santé b) Conseils pratiques : apprentissage de techniques pédagogiques pour rendre les enfants plus actifs dans le contexte scolaire et diminuer le temps sédentaire, inclusion des savoirs liés aux comportements de santé dans l'ensemble des matières académiques (interdisciplinarité). c) Formation aux techniques de relaxation (sophrologie) afin de favoriser le bien-être des enfants et diminuer le temps sédentaire. 
Les enseignants ont également reçu un livret avec différents exercices de relaxation. Ils ont également été encouragés à être actifs et ont porté également des capteurs pour connaître leurs niveaux d'activité physique et de sédentarité.

Au cours de la présentation, des enseignants ayant vécu cette intervention au sein des écoles seront présents pour témoigner. Ils feront part de leurs connaissances, idées et expériences vécues.

Prochainement, des analyses statistiques seront réalisées, afin de tester :

1. L'efficacité de l'intervention AP et ST à l'école

2. Les liens entre activité physique, sédentarité, capacités attentionnelles et réussite scolaire.

\section{Conclusion :}

A la suite de cette étude, il est prévu d'améliorer les programmes d'intervention afin de les proposer à d'autres écoles en lien avec les services d'éducation de la région, le but étant de sensibiliser une plus grande partie des élèves. Cette étude, dont le but est de promouvoir l'activité physique chez les enfants, souhaite engendrer des connaissances et savoir-faire chez cette population qui les feront adopter des bonnes habitudes en termes de santé qui s'accompagneront d'une augmentation de leur bien-être au cours de toute leur vie.

\section{Bibliographie :}

Barnes, J., Behrens, T. K., Benden, M. E., Biddle, S., Bond, D., Brassard, P., ... \& Colley, R. (2012). Letter to the Editor: Standardized use of the terms "sedentary" and "sedentary behaviours". Applied Physiology Nutrition and Metabolism-Physiologie Appliquee Nutrition Et Metabolisme, 37(3), 540-542.

Bronfenbrenner, U. (1977). Toward an experimental ecology of human development. American psychologist, 32(7), 513-531.

Donnelly, J. E., Hillman, C. H., Castelli, D., Etnier, J. L., Lee, S., Tomporowski, P.,... \& Szabo-Reed, A. N. (2016). Physical activity, fitness, cognitive function, and academic achievement in children. Medicine \& Science in Sports \& Exercise, 48(6), 1197-1222. doi: 10.1249/mss. 0000000000000901

Grélot, L. (2016). Activités physiques et sportives de l'enfant et de l'adolescent : des croyances aux recommandations sanitaires. Journal de Pédiatrie et de Puériculture, 29(2), 57-68. doi: 10.1016/j. jpp.2016.02.003 


\section{Les « breaks » de sédentarité Organisation de la classe pour diminuer le temps sédentaire Activités « actives » dans la classe}

Caroline Bernal ${ }^{1}$ y Nicolas Fabre ${ }^{1}$

${ }^{1}$ Université de Pau

\section{Résumé :}

L'école traditionnelle classique impose aux enfants de rester en classe assis afin d'acquérir de nouvelles connaissances et de nouvelles compétences. Ce temps passé assis en classe peut être évalué à 5 heures voir plus, selon les horaires des établissements.

Or, l'accumulation de temps en position sédentaire aurait des effets négatifs sur la santé, et ce, dès la jeune enfance. II est donc primordial de limiter autant que possible le temps passé en position sédentaire et de montrer aux enfants comment limiter ce temps assis.

L'enfant apprend par les expériences vécues, et notamment par les mouvements réalisés. II est donc justifié d'employer des méthodes qui rendent les enfants actifs dans leurs apprentissages et dans leur vie, les menant à expérimenter.

Comment diminuer le temps sédentaire en classe et rendre les enfants actifs dans leurs apprentissages?

Afin de diminuer le temps sédentaire en classe, plusieurs stratégies sont possibles et complémentaires. Tout d'abord, nous verrons que l'enseignant peut effectuer des coupures, des « breaks » ou l'enfant va être actif pendant une dizaine de minutes en classe, mais nous verrons également que l'enseignement peut adapter l'organisation et les méthodes d'apprentissages utilisées avec sa classe pour diminuer ce temps passé assis.

1. Effectuer des «breaks » de sédentarité en classe dans les moments ou les enfants cumulent trop de temps en position sédentaire

1.1. Effectuer une pause dans les apprentissages en cours afin d'exercer des exercices de respiration, de relaxation, de sophrologie

Beaucoup d'adultes travaillent assis en position sédentaire (assis). Nous leurs conseillons de se lever toutes les heures et de s'étirer, de marcher, afin d'éviter les effets négatifs de la sédentarité. A l'école, les enfants restent également assis. Le principe des pauses actives pour les enfants repose sur le même principe que pour les adultes. Lors d'une longue phase passée en position assise, les enseignants vont décider de faire un break 
de 10 minutes pendant le cours afin de réaliser des exercices de respiration, relaxation, sophrologie (appelés « routines de pratiques corporelles de bien-être »). Ces coupures permettent non seulement aux enfants de bouger et de diminuer le temps sédentaire, mais aussi de se ressourcer avant de rependre le fil des apprentissages : les apprentissages liées au cours de français, mathématiques, etc... sont laissés de côté pendant la pause active.

Cependant, ces pauses actives doivent inclure des exercices physiques à allure faible ou modérée pour être efficaces. En effet, si l'intensité d'effort est trop importante lors de ces pauses, elles sont contre-productives pour les apprentissages et pour la réussite scolaire des enfants $(\mathrm{Hi}-$ IIman \& Castelli, 2009). Une intensité trop importante amène de la fatigue et une réduction des performances cognitives.

Ces pauses actives sont réalisées dans de nombreux pays. Le programme Energizers " Activity Promotion Laboratory ", mené aux USA (Mahar et al., 2006), a montré que les enfants qui réalisaient des pauses actives pendant un cours passaient plus de temps concentré et à travailler sur une tâche, comparativement aux enfants qui n'avaient pas de pauses actives.

\subsection{Effectuer des ateliers de français, mathématiques, etc... en faisant bouger les élèves}

Les pauses actives vu précédemment laissent de côté les apprentissages liés aux matières académiques qui étaient alors abordées pour faire place à ce moment d'activité. Plusieurs études se sont alors intéressées aux effets d'exercices physiques réalisées en même temps que les apprentissages. Le cours de mathématique ou de français va donc se poursuivre pendant la pause active, mais tout en faisant bouger les élèves. Certaines classes utilisent des ballons de fitness («Swiss-balls » comme support pour être assis), ou encore des tapis de course: cependant, les effets de l'utilisation de ce matériel pendant l'acquisition des connaissances et compétences sont toujours en questions. De plus, il y a peu d'information concernant les effets positifs sur la lecture lorsque les élèves pédalent sur un vélo d'appartement lorsque la leçon de lecture est réalisée en même temps, chez les enfants d'école primaire (Hillman \& Catelli, 2009).

Ces matériaux (vélo, tapis de course, etc...) imposent aux enfants une pratique mécanique de l'activité physique, sans aspect ludique. Dans ce cas, l'enfant risque de ne pas savoir gérer son intensité d'effort, ou de se disperser dans les multiples taches à effectuer, pouvant être au détriment de ses apprentissages. De plus, cet aspect mécanique de l'activité physique ne l'incite pas à être actif en dehors de ce programme.

Des programmes ont donc été établis afin de réaliser des pauses actives pendant les cours, mais tout en incluant un aspect ludique à ces pauses. Le programme TAKE 10 (Stewart et al., 2004) a été mené aux USA auprès de 50000 classes et implique plus de 1 million d'enfants. Le but de ce 
programme était d'inclure des pauses actives dans les matières scolaires, tout en continuant à travailler sur le cours en effectuant des mouvements.

Voici plusieurs exemples concrets réalisés dans ce programme :

- Les élèves effectuent 10 sauts pour chaque réponse correcte effectuée. Pendant le cours de mathématiques, les enfants sautent tous les 5 unités (5-10-15-20), toutes les deux unités (2-4-6-8) de manière croissante ou décroissante.

- Pour l'apprentissage des mots et leur orthographe, les élèves peuvent toucher différentes parties du corps lorsque les mots sont épelés (CHAT : $\mathrm{C}$ on touche la tête ; $\mathrm{H}$ on touche les épaules ; $\mathrm{A}$ les genoux; $T$ les pieds).

- Lorsque l'on raconte une histoire ou lorsque l'on lit un livre, les élèves peuvent être en mouvement sur les mots d'action dans l'histoire (promenade $=$ on va se promener ; taper des mains $=$ on tape des mains). On peut aussi expliquer des notions compliquées aux enfants via les mouvements.

Ce programme a un impact positif sur la réussite scolaire des enfants d'école primaire (Donnelly \& Lambourne, 2011), temps passé à travailler sur la tâche (Goh, Hanon, Webster, \& Hannon, 2016) ainsi que sur leur comportement (Ribeiro \& Alves, 2014).

2. Méthodes pédagogiques amenant les élèves à se déplacer dans la classe

\subsection{Organisation d'ateliers}

Dans l'organisation de classe, il y a la possibilité de réaliser plusieurs ateliers à différentes tables, ou les enfants sont debout et vont expérimenter dans chacun des ateliers ; lorsqu'ils ont fini un atelier, ils permutent d'ateliers et sont ainsi en mouvement. A la fin des ateliers, les élèves rangent le matériel. Ce principe est utilisé dans la pédagogie Montessori. Montessori fait des ateliers ou les enfants vont expérimenter, apprendre par leurs propres gestes et associe donc le mouvement à la pensée. De plus, l'espace de la classe est géré afin que les élèves puissent circuler librement entre les ateliers. L'enfant va travailler sa coordination motrice dans l'ambiance d'ateliers crée par l'adulte, lui permettant d'atteindre des buts qui ont du sens pour lui. Maria Montessori considère que " c'est par l'action que l'esprit arrive à s'élever ", attribuant une place fondamentale au mouvement. Selon elle, l'enfant doit développer son corps en même temps que son esprit. Les enfants utilisent leurs muscles, leurs mains, leurs pieds pour explorer le monde. Les enfants ne peuvent pas grandir dans l'immobilité et il n'est pas nécessaire de se tenir dans une position spéciale pour profiter de l'éducation ou pour apprendre. La coordination des 
muscles doit être perfectionnée par le développement cognitif : ces idées rejoignent les activités réalisées dans les pauses actives ou l'on travaille les différentes matières académiques en mouvement.

\subsection{Organisation dans la vie commune.}

Afin de diminuer le temps sédentaire, il peut être également intéressant de donner à faire des tâches actives aux enfants, leur permettant de leur donner des responsabilités. Par exemple, toutes les semaines, un élève a pour objectif de s'occuper de distribuer les photocopies, un autre élève a comme rôle de faire les photocopies pendant cette même semaine, un autre enfant peut aller voir les données météo dans la cour tous les matins, etc... ainsi, les enfants ont des tâches actives à réaliser. Cette organisation de classe est notamment utilisée dans la pédagogie Freinet. Les enfants ont des tâches individualisées mais participent également à l'œuvre collective du groupe.

En conclusion, plusieurs méthodes sont possibles afin de diminuer le temps sédentaire des enfants en classe. Toutes ces méthodes sont complémentaires et peuvent ainsi permettre aux enseignants de varier leur approche pédagogique avec les élèves.

\section{Bibliographie :}

Castelli, D. M., \& Hillman, C. H. (2012). Physical activity, cognition, and school performance: from neurons to neighborhoods. In Physical Activity Across the Lifespan (pp. 41-63). Springer New York.

Donnelly, J. E., \& Lambourne, K. (2011). Classroom-based physical activity, cognition, and academic achievement. Preventive medicine, 52, S36-S42.

Freinet, C. (1973). Les techniques Freinet de l'école moderne. Paris: A. Colin, coll. Bourrelier.

Goh, T. L., Hannon, J., Webster, C., Podlog, L., \& Newton, M. (2016). Effects of a TAKE 10! Classroom-Based Physical Activity Intervention on Third-to Fifth-Grade Children's On-task Behavior. Journal of Physical Activity and Health, 13(7), 712-718.

Mahar, M. T., Murphy, S. K., Rowe, D. A., Golden, J., Shields, A. T., \& Raedeke, T. D. (2006). Effects of a classroom-based program on physical activity and on-task behavior. Medicine and science in sports and exercise, 38(12), 2086.

Montessori, M., \& Bernard, G. (2016). La découverte de l'enfant: Pédagogie scientifique (Vol. 1). Desclée de Brouwer.

Ribeiro, R. Q., \& Alves, L. (2014). Comparison of two school-based programmes for health behaviour change: the Belo Horizonte Heart Study randomized trial. Public health nutrition, 17(6), 11951204. 


\section{Exemple de mise en place du travail collaboratif}

Pascal Legrain ${ }^{1}$ Guillaume Escalie ${ }^{1}$

'UF STAPS, Université de Bordeaux

\section{Résumé de l'atelier :}

Cet atelier consiste à faire vivre à l'audience un dispositif d'apprentissage coopératif de type Jigsaw (Aronson \& Patnoe, 1997). Le principe pédagogique sous-jacent à cette démarche consiste à considérer que l'expérimentation de dispositifs coopératifs d'apprentissage en formation par les enseignants peut favoriser leur mise en œuvre ultérieure en classe avec les élèves.

Le dispositif Jigsaw ("Groupe d'Experts ") présente les caractéristiques distinctives suivantes. Les acteurs sont placés en équipe. Le matériel ou les ressources sont divisés en sous-parties invitant les membres de chaque équipe à se répartir sur divers ateliers pour accéder efficacement à l'intégralité des ressources. Dans un premier temps, chaque membre a la responsabilité d'apprendre une partie de la tâche au sein d'un groupe dit " d'experts ". Dans un second temps, il lui est demandé de retourner dans son équipe d'origine pour enseigner à ses co-équipiers ce qu'il a appris, et apprendre de ceux-ci les informations qu'ils produisent.

L'atelier prend appui sur l'activité physique Savate Boxe Française. Les participants, placés en équipes de huit, rejoignent par deux un atelier d'expertise portant sur l'acquisition d'une technique de pied (directo u crochet) ou de poing (chassé ou fouetté). Dans un premier temps, les participants se voient instruits par une vidéo enregistrée sur une tablette sur la technique qu'ils doivent s'approprier. Dans un deuxième temps, les participants, appareillés en dyades, pratiquent cette technique, Dans un troisième temps, le formateur mène une activité d'observation-régulation amenant les participants à s'interroger sur les difficultés majeures d'apprentissage rencontrées dans leurs ateliers respectifs. Enfin, il leur est demandé de rejoindre leur équipe pour enseigner en qualité de tuteur les connaissances et habiletés acquises, et pour apprendre en qualité de tutoré, les connaissances et les habiletés que leurs partenaires d'équipe leur enseignaient. L'objectif collectif final de l'équipe consiste en ce que chaque membre soit capable de produire un enchaînement intégrant les quatre techniques abordées.

\section{Bibliographie :}

Aronson E. \& Patnoe S. (1997). The jigsaw classroom: Building cooperation in the classroom. New York: Addison-Wesley.

Escalié, G., Legrain, P., \& Lafont, L. (2018). L'apprentissage coopératif en " groupe d'experts » et la professionnalisation des futurs enseignants: un exemple en Education Physique et Sportive. Carrefours de l'éducation, 46, 161-176.

Legrain, P. (1998). Boxe française. Apprentissage et enseignement. Paris: Editions Revue EPS (coll. Les cahiers du sport). 


\title{
Théorie et pratique sur la promotion d'AP : Rencontres inter-centres. "Le Test de Responsabilité”. Une stratégie avant les Rencontres inter-centres
}

\author{
Paz Tierz', Gemma Bermejo ${ }^{2}$, Raquel Pérez-Ordás ${ }^{2}$ y Eduardo Generelo-3 \\ 1 Instituto de Educación Secundaria Sierra de Guara. Huesca. \\ 2 CAPAS-Ciudad Huesca. \\ 3 Facultad de Ciencias de la Salud y del Deporte. Universidad de Zaragoza.
}

\section{Résumé :}

Le stage présente et expose le " test de responsabilité ». II s'agit d'une situation de travail qui utilise cinq jeux coopératifs afin de stimuler la capacité d'organisation et de coopération chez les élèves adolescents pour résoudre des problèmes en groupe. Le test constitue une étape à franchir dans le processus complexe de responsabilisation des sujets afin qu'ils deviennent les acteurs de leur propre pratique d'activité physique. Les Rencontres inter-centres sont une situation de référence dans ce processus.

\section{Introduction et contextualisation :}

Nous souhaitons que les groupes scolaires soient capables de participer avec le plus d'autonomie possible à la vie de l'établissement. Organiser les récréations, participer à des commissions, intervenir dans la résolution de conflits, contribuer à la gestion de la bibliothèque, organiser et participer à des activités sportives et récréatives, etc. sont autant d'actions qui constituent un apprentissage de base orienté vers l'objectif général de formation de citoyens participatifs et critiques dans la société où ils vivront.

Le " test de responsabilité » est un outil qui sert à orienter les actions visant à ce qu'un groupe scolaire s'implique et endosse des responsabilités dans l'activité scolaire.

Le " test de responsabilité » est une proposition qui est réalisée comme un défi lancé à un groupe scolaire qui doit le résoudre en un temps déterminé. L'équipe de professeurs et les élèves observent le résultat du test et se basent sur ce dernier et sur le processus effectué par le groupe pour encadrer depuis le débat les aptitudes sociales et les règles de comportement en groupe qui leur permettront d'affronter efficacement les problèmes pour lesquels la collaboration sera essentielle.

Participer à l'organisation des récréations, aux Rencontres inter-centres ou à des voyages scolaires sont par exemple des situations de référence pour lesquelles il faut déployer tout un processus où le test de responsabilité représente un point d'appui. 


\section{Description de la proposition :}

Le test consiste à proposer cinq épreuves à un groupe d'élèves avec la collaboration de toute la classe. Les objectifs réels que les enseignants recherchent sont les suivants :

1. Connaître le comportement d'un groupe qui affronte un problème de manière collective.

2. Identifier le rôle que chaque membre joue dans le groupe.

3. Faire prendre conscience au groupe des objectifs précédents.

4. Orienter les différentes aptitudes sociales qui sont essentielles pour aborder des actions collectives.

Le processus uni au test de responsabilité entraîne une série d'actions de la part des enseignants et des élèves qui se décompose comme suit :

1. Réalisation du test initial.

2. Travail coopératif en classe pour analyser le déroulement du test.

3. Répétition du test.

4. Détermination des étapes à suivre pour résoudre les problèmes posés lors de l'exécution du test. Définir des codes liés aux aptitudes sociales et collectives.

5. Application des stratégies convenues afin de responsabiliser les élèves et motiver leur autonomie pour déclencher des actions propres (Rencontres inter centres, récréations amusantes, sorties, etc.)

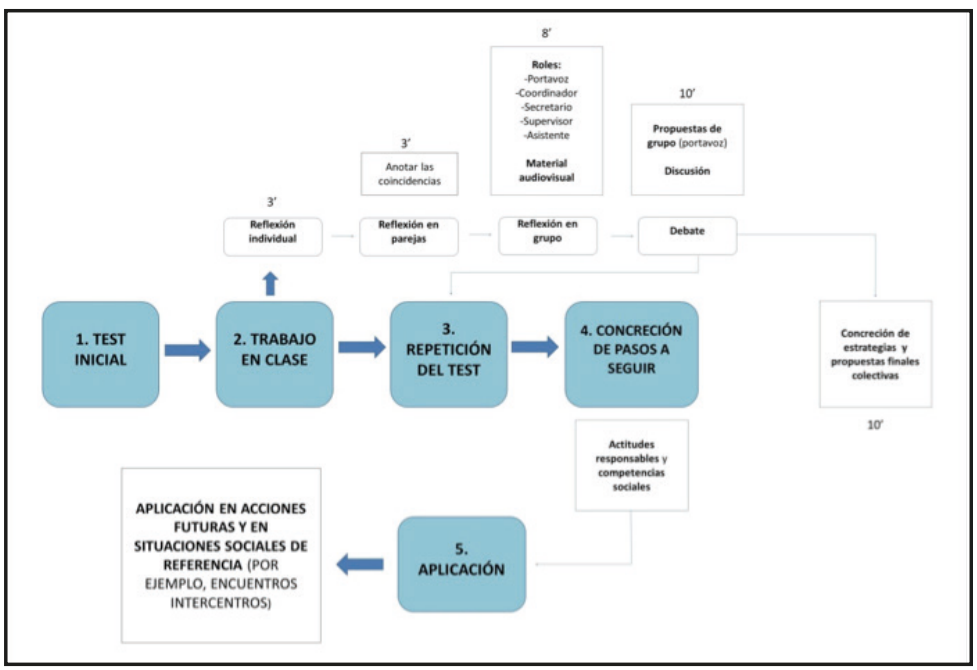




\section{Situations clés d'apprentissage :}

Le processus de travail consiste à lancer un défi au groupe, vérifier si l'objectif du jeu est atteint et analyser avec le groupe les raisons qui ont permis de réussir ou pas. Le test doit se dérouler en plusieurs séances de travail et il est essentiel que le suivi soit effectué par un même professeur ou une même équipe d'enseignants dans la cadre d'une proposition d'intervention qui revêtira si possible un caractère transversal.

\section{Conclusion :}

Le " test de responsabilité " représente un outil très intéressant pour favoriser la cohésion du groupe et le développement de l'autonomie dans le travail pour participer à un modèle comme les Rencontres inter-centres.

\section{Bibliographie :}

Fraile, A. (2003). Actividad física y salud. Educación secundaria. Valladolid: Junta de Castilla y León.

Murillo, B., Sevil, J., Julián, J. A., \& Generelo, E. (2018). Sigue la Huella: Guide à l'usage des enseignants pour la promotions d'habitudes de vie saínes chez les élèves. CAPAS-Cité.

Santed, M. (2016). El valor del juego cooperativo en alumnos de $2^{\circ}$ de ESO. Trabajo Fin de Máster. Universidad de Zaragoza, Huesca. 


\section{Théorie et pratique sur la promotion de l'AP: Rencontres inter-centres}

José Antonio Julián ${ }^{1-2}$, Javier Sevil' ${ }^{2}$, Alberto Aibar ${ }^{2-3}$

1 Facultad de Ciencias Sociales y Humanas. Universidad de Zaragoza.

2 Grupo de Investigación EFYPAF. Universidad de Zaragoza.

3 Facultad de Ciencias Humanas y de la Educación. Universidad de Zaragoza.

\section{Résumé:}

Cet atelier aborde la mise en pratique de l'activité physique et sportive du volley-ball comme contenu de l'éducation physique et sportive scolaire au moyen d'une nouvelle proposition d'enseignement-apprentissage appelée Smashball (http://es.smashbal.nl/). Cette proposition vise à faire participer les étudiants à une situation sociale de référence scolaire de la ville de Huesca qui s'intitule « Rencontre inter-centres de volley-ball ».

\section{Introduction et contextualisation :}

La ville de Huesca propose une série de rencontres sportives inter-centres auxquelles participent tous les établissements de l'enseignement secondaire de la ville de Huesca. Ces rencontres qui présentent un caractère ludique et festif souhaitent devenir des situations sociales de référence pour la construction de processus d'enseignement-apprentissage de contenus d'éducation physique et sportive (EPS) scolaire. Les enseignants utilisent tout le potentiel éducatif de ces rencontres en les incluant dans leurs programmes et en proposant les unités didactiques correspondantes dans les semaines avant la réalisation de ces rencontres. Un de ces événements auxquels les élèves de $2^{\circ}$ de ESO (4ème) de la ville de Huesca participent est un tournoi de volley-ball $3 \times 3$. La réflexion méthodologique de l'ensemble des enseignants sur l'enseignement avant le volley-ball afin de pouvoir favoriser une meilleure participation de tous les élèves, est donc fondamentale pour une construction sociale du sport en tant qu'activité inclusive et intégratrice.

\section{Description de la proposition :}

Le volley-ball est une activité qui habituellement a été enseignée à partir d'une approche plus traditionnelle, la complexité de certains gestes techniques ne facilitant pas parfois son enseignement au niveau scolaire. Une telle caractéristique génère des difficultés pour l'élaboration de propositions éducatives où tous les élèves peuvent se sentir compétents et réussir la pratique. Le smashball est une forme jouée de volley-ball qui a été créée récemment par la Fédération hollandaise afin d'assouplir les règles et le 
dynamisme de l'activité au moyen de l'introduction du smash qui constitue le principal élément moteur du jeu. L'organisation de son enseignement autour de cet aspect du jeu cherche à générer une pratique sportive plus inclusive, participative et accessible à tous les élèves.

\section{Situations clés d'apprentissage :}

Ce stage présente une série de situations d'apprentissage initiales du Smashball qui sont plus orientées vers les cycles d'éducation primaire. Trois situations d'apprentissage du Smashball (N1, N2, N3) sont notamment proposées pour répondre à certains aspects principaux de l'enseignement du volleyball.

1. Smashball N1: situation initiale de $2 \times 2$ qui présente la notion de Smash (le joueur se lance le ballon lui-même et frappe) comme élément fondamental de jeu et solution aux difficultés techniques généralement posées par le volley-ball. Dans cette situation, les joueurs peuvent attraper le ballon avec ou sans rebond et peuvent progresser avec.

2. Smashball N2 : situation $2 \times 2$ pour progresser dans la construction du jeu en introduisant d'autres options de Smash (interaction avec un coéquipier) et à partir duquel il faut chercher à améliorer la technique du smash pour plus d'efficacité dans le jeu. L'interaction entre les coéquipiers se fait par des touches assistées.

3. Smashball N3 : situation $2 \times 2$ pour progresser dans la construction du jeu en exigeant l'introduction d'une frappe dans la passe au coéquipier. Cette frappe entraîne le besoin d'introduire d'autres actions qui favorisent l'apprentissage de certains gestes techniques comme la passe avec les dix doigts et le service à la cuillère.

Il est recommandé de consulter le document de Domingo \& Julián (2017) pour obtenir plus d'informations sur la description d'autres situations plus appropriées à l'enseignement secondaire et d'aspects généraux de la séquence didactique de l'enseignement du Smashball.

\section{Conclusion :}

Le Smashball est une proposition d'enseignement-apprentissage du volley-ball dont la proposition s'adapte parfaitement à la réalisation de rencontres inter-centres où prédomine une ambiance ludique favorisant la réussite de tous les participants. Cette proposition prétend favoriser une EPS de qualité. 


\section{Bibliographie :}

Domingo, J., \& Julián, J. A. (2017). Secuencia didáctica para la introducción del voleibol-smashball en el contexto escolar: Una propuesta metodológica a los deportes de cancha dividida en contexto CRA. IV Jornadas de Educación Física +EF. Almudévar (Huesca). 25 de noviembre de 2017. 


\title{
Réflexions sur l'organisation des situations d'enseignement en E.P.S. ayant un impact sur la santé : Le travail intermittent à haute intensité en sports collectifs
}

\author{
Laurent Souriac ${ }^{1}$ \\ 1'Département Staps Tarbes, Collège SSHS, \\ Université de Pau et des Pays de l’Adour
}

\section{Résumé :}

Mieux organiser les situations d'enseignement est une piste de réflexion sur laquelle les enseignants se penchent depuis longtemps pour augmenter le temps moteur des élèves en EPS. Les travaux récents sur les exercices intermittents à haute intensité (HIIE) viennent apporter un éclairage différent et complémentaire qu'il faut considérer avec intérêt. Cet atelier a pour but, à partir d'un contexte réel d'enseignement de l'EPS en Basket-ball, de réfléchir à la fois à l'augmentation du temps d'activité physique dans un cours d'EPS et à un meilleur ciblage des formes d'activités et de leurs impacts.

\section{Présentation :}

Les préoccupations liées à la santé dépassent largement les pratiques de la CP5. Toutes les APSA scolaires peuvent y contribuer, notamment les sports collectifs (Handball et Basket-ball), car ils sont encore un pan important des pratiques d'enseignement scolaire. Il convient alors de s'interroger sur les mises en œuvre didactiques les plus favorables au cours de la leçon.

Lors de l'échauffement et de situations d'apprentissages ciblés sur certaines capacités (passes, rebonds, dribbles, doubles pas, tirs, plaquages...) le travail sous forme de parcours est très souvent utilisé et intéressant car il réduit les temps d'attente.

Lors de situations à valences tactiques, le travail collectif à effectifs réduits et sur des aspects de jeu particuliers est privilégié (tous les types de 3c2, 4c3, combinaisons, soutien défensif...). Dans ce cas, ce sont des ateliers spécifiques, plus ou moins différenciés, qui sont mis en jeux pour optimiser les temps de pratique.

Enfin, lors des situations à valences stratégiques sur des effectifs com- 
plets et incluant de la réversibilité la forme traditionnelle est la situation de match. Ces moments sont particulièrement problématiques car ils sont complexes à gérer et la plupart du temps ils sont synonymes de temps d'inactivité (beaucoup d'élèves et peu de terrains).

Dans cet atelier de 12 pratiquants pour un petit terrain de $20^{*} 12$, nous allons pouvoir comparer 3 situations stratégiques différentes.

- 2 matchs de basket-ball en 3c3 de 3 minutes: 6 joueurs inactifs sur 3 min, des Fréquences cardiaques ( $\mathrm{Fc}$ ) qui sont sous maximales.

- 1 série de 6 minutes d'attaques défenses en 3c3 (l'équipe attaque puis défend puis sort pour laisser sa place à l'équipe en attente). Dans ce cas, nous optimisons le temps de pratique, tous les élèves sont en jeu sur 6 minutes, ils sont plus impliqués d'un point de vue informationnel. Cette implication permet de fixer les élèves sur la tâche plus longtemps, permettant de réduire les temps d'activités déviantes. D'un point de vue énergétique, le travail n'est plus de forme continue mais intermittente ce qui a pour conséquence d'augmenter le temps passé à des Fc supérieures car la récupération est incomplète.

- 1 série de 6 minutes d'attaques-défenses en 3c3 à haute intensité. Selon la même organisation, des plots sont disposés sur les côtés du terrain. Dès qu'une équipe doit défendre les joueurs sont retardés car ils doivent contourner les plots par l'extérieur (sortie du terrain). Ce retard de la défense a pour conséquence d'accélérer fortement la vitesse de l'attaque (puisque la zone à attaquer est presque libre). On a ici un jeu à égalité numérique basé sur un enchaînement de contre-attaques et de repli-défensifs rapides. Ce travail associe à l'optimisation du temps d'activité motrice une intensité de pratique se rapprochant ou dépassant les capacités aérobies maximales (travail à PMA). De nombreux travaux nous montrent l'impact très positif de ce type de travail.

Même si l'on ne contrôle pas les vitesses des joueurs, on se rapproche fortement d'un protocole de travail en 15 "- 15 " se situant entre $100 \%$ et $120 \%$ de la VMA avec des valeurs proches des Fc max. Le tableau suivant permet de vérifier le profil des élèves qui peuvent en bénéficier. Si les élèves ont des VMA supérieures le travail peut s'effectuer sur grand terrain de BB $(28 \mathrm{~m})$ (lycée par exemple). Dans un contexte réel d'enseignement, il est possible de réguler le rapport de force en limitant ou en supprimant le retard de certains joueurs qui sont plus en difficulté. 


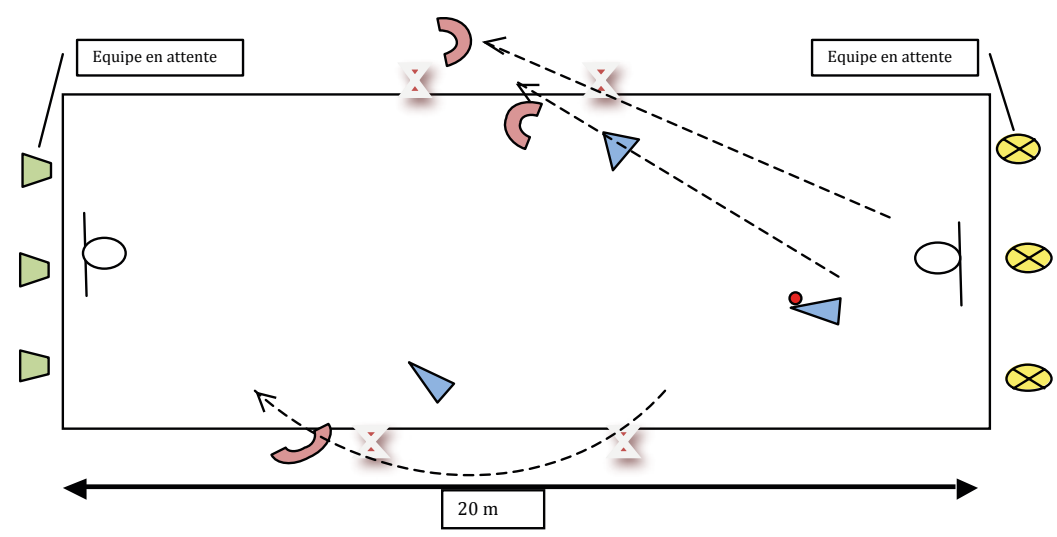

Ce travail présenté en Basket-ball en 3c3 a été testé en BB en 4c4, et en handball en $4 c 4,5 c 5$ et $6 c 6$. L'impact du travail peut-être évalué en associant l'utilisation d'Echelles RPE (Borg, 1970) ou ETL (Garçin et al., 1999).

\begin{tabular}{|c|c|c|c|c|c|c|c|c|c|}
\hline \multicolumn{10}{|c|}{ Exemple de durées d'attaque-défense en BB pour une classe de cycle 4 en fonction de la VMA sur petit terrain } \\
\hline VMA en $\mathrm{km} / \mathrm{h}->$ & 8 & 9 & 10 & 11 & 12 & 13 & 14 & 15 & 16 \\
\hline adaptations sco tps $40 \mathrm{~m}$ & 4,4 & 5,0 & 5,6 & 6,1 & 6,7 & 7,2 & 7,8 & 8,3 & 8,9 \\
\hline $100 \%$ VMA & 18,0 & 16,0 & 14,4 & 13,1 & 12.0 & 11,1 & 10,3 & 9,6 & 9,0 \\
\hline $110 \%$ VMA & 16,4 & 14,5 & 13,1 & 11,9 & 10,9 & 10,1 & 9,4 & 8,7 & 8,2 \\
\hline $120 \%$ VMA & 15,0 & 13,3 & 12,0 & 10,9 & 10,0 & 9,2 & 8,6 & 8,0 & 7,5 \\
\hline $1309 \mathrm{VMMA}$ & 13,8 & 12,3 & 11,1 & 10,1 & 9,2 & 8,5 & 7,9 & 7,4 & 6,9 \\
\hline
\end{tabular}

\section{Bibliographie :}

Bond, B., Weston, K. L., Williams, C. A., \& Barker, A. R. (2017). Perspectives on high-intensity interval exercise for health promotion in children and adolescents. Open access journal of sports medicine, 8, 243-265.

Borg G. (1970). Perceived exertion as an indicator of somatic stress. Scand J Rehabil Med, 2(2), 92-98.

Garcin, M., Vandewalle, H., \& Monod, H. (1999). A new rating scale of perceived exertion based on subjective estimation of exhaustion time : A preliminary study. Int J Sports Med, 20(1), 40-43. 


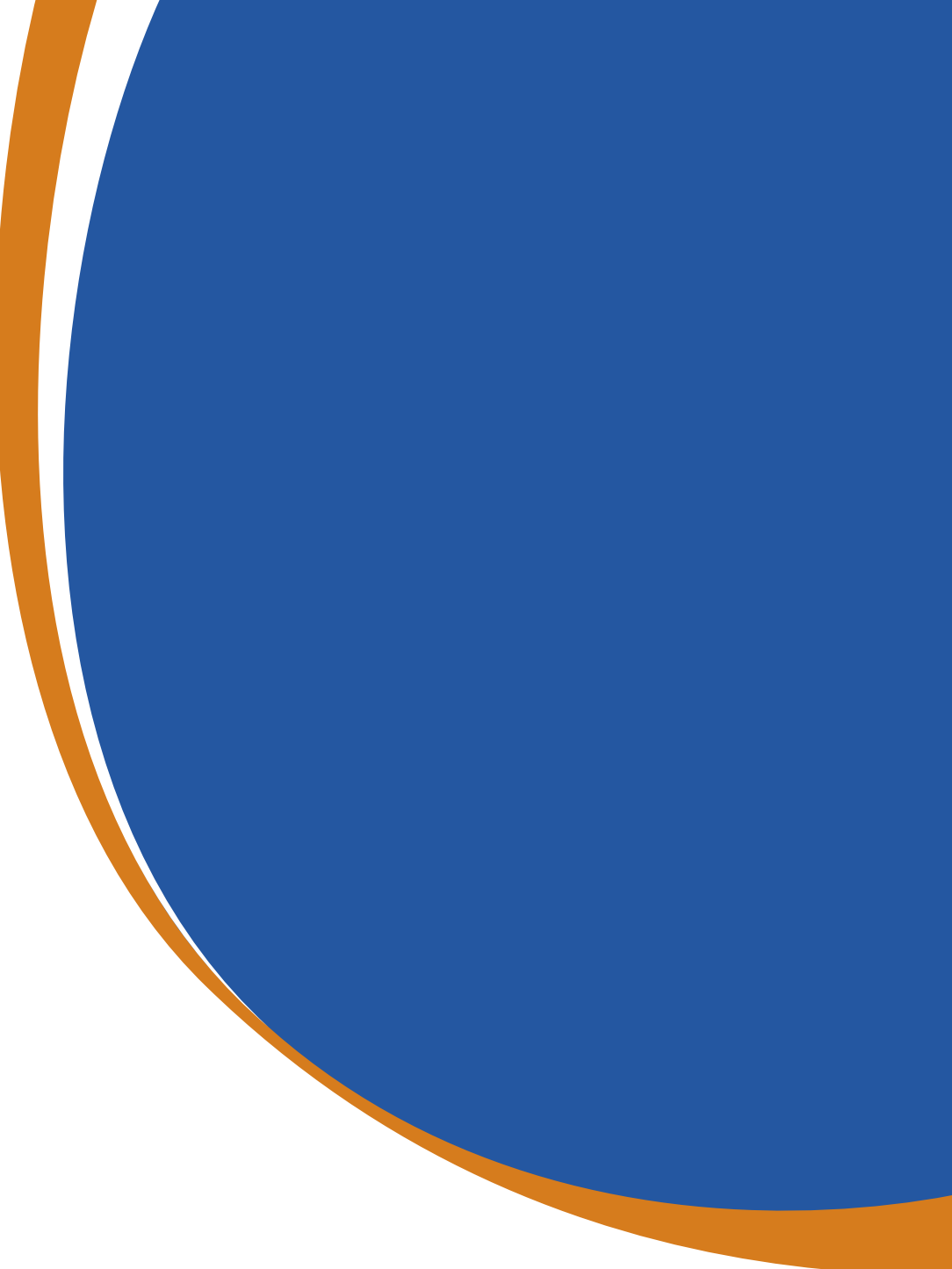

